\title{
Blocking of Lung Endothelial Cell Adhesion Molecule-1 (Lu-ECAM-1) Inhibits Murine Melanoma Lung Metastasis
}

\author{
Duzhang Zhu, Chao-Fu Cheng, and Bendicht U. Pauli \\ Cancer Biology Laboratories, Department of Pathology, Cornell University College of Veterinary Medicine, Ithaca, New York 14853
}

\begin{abstract}
The 90-kD lung endothelial cell adhesion molecule-1 (LuECAM-1) selectively promotes $\mathrm{Ca}^{2+}$-dependent adhesion of lung-metastatic B16 melanoma cells. Corresponding with their metastatic performance, high lung-metastatic B16-F10 melanoma cells bind in significantly higher numbers to Lu-ECAM-1 than their intermediate and low lung-metastatic counterparts B16-L8-F10 and B16-F0, respectively. Maximum attachment is observed at a density of $\sim 2.4 \times 10^{2} \mathrm{Lu}-\mathrm{ECAM}-1$ sites $/ \mu \mathrm{m}^{2}$ of plastic surface. B16 melanoma cell binding to Lu-ECAM-1 is blocked by mAb 6D3 and is competitively inhibited by soluble Lu-ECAM-1. C57Bl/6 mice passively immunized with anti-Lu-ECAM-1 mAb 6D3 or actively immunized with purified Lu-ECAM-1 exhibit an anti-Lu-ECAM-1 antibody titerdependent reduction in the number of B16 experimental metastases. Lu-ECAM-1 promotes neither binding nor metastasis of other lung-metastatic tumor cells (e.g., KLN205). Our data indicate that an "antiadhesion" therapy directed at interfering with the adherence of blood-borne tumor cells to organ-specific vascular endothelium is efficient in the control of metastasis formation in selective organ sites. (J. Clin. Invest. 1992. 89:1718-1724.) Key words: lung metastasis • endothelial cell adhesion molecule • B16 melanoma cells • organ specificity • monoclonal antibodies $\bullet$ adhesion inhibition
\end{abstract}

\section{Introduction}

Adhesion molecules expressed by vascular endothelial cells have been implicated with the arrest of blood-borne cancer cells in selected secondary organ sites and the initiation of extravasation (1-6). These endothelial cell adhesion molecules (ECAMs) $^{1}$ are part of an organ-specific, constitutive vascular phenotype that appears to be regulated through complex interactions with components of the extracellular matrix $(7,8)$. Similar to the adhesion molecules of high endothelial venules that direct the homing of lymphocytes to selective lymphoid tissues (9-11), organ-specific ECAMs serve as attachment sites for

Address correspondence and reprint requests to Dr. Bendicht U. Pauli, Cancer Biology Laboratories, Department of Pathology, Cornell University College of Veterinary Medicine, Ithaca, NY 14853.

Received for publication 20 August 1991 and in revised form 18 December 1991.

1. Abbreviations used in this paper: BAEC, bovine aortic endothelial cells; ECAMs, endothelial cell adhesion molecules; FBS, fetal bovine serum; Lu-ECAM-1, lung-specific endothelial cell adhesion molecule-1.

J. Clin. Invest.

(c) The American Society for Clinical Investigation, Inc.

$0021-9738 / 92 / 06 / 1718 / 07 \quad \$ 2.00$

Volume 89, June 1992, 1718-1724 blood-borne cancer cells and, thus, determine the tumor-specific metastatic organ colonization patterns known for many years by clinical oncologists (12-14). Using a unique large-vessel endothelial cell system in which an organ-specific vascular phenotype can be induced by growing "neutral" bovine aortic endothelial cells (BAEC) on matrix extracts of that organ (7), a lung-specific endothelial cell adhesion molecule (Lu-ECAM-1) has recently been isolated by our laboratory $(6,15)$. A monoclonal antibody (mAb 6D3) developed against this molecule, cross-reacts with a murine homologue expressed on the endothelial cell lumenal membrane of selected branches of mouse pulmonary veins. Lu-ECAM-1 selectively promotes the attachment of lung-metastatic B16 melanoma cells and mediates metastatic colonization of the lungs by these tumor cells (6).

The critical dependence of blood-borne cancer cells upon organ-specific endothelial cell adhesion molecules in metastasis may provide a unique opportunity of interfering with the metastatic cascade. The concept of interfering with cell-cell adhesion phenomena to achieve a therapeutic advantage has recently been introduced to combat excessive leukocyte participation and associated tissue damage in acute and chronic allergic, autoimmune, and inflammatory diseases (16-18). Many of the published reports have used a mAb directed against CD18 that prevents binding of leukocytes via its LFAl or Macl receptors to endothelial cells. This mAb has a remarkable ability to prevent various forms of neutrophil-mediated ischemia-reperfusion damages in rabbits when administered at the time of perfusion. Therapeutic blocking of the endothelial cell rather than the leukocyte adhesion partner has been demonstrated in a model of bronchial asthma in which airway eosinophilia and hyperresponsiveness are attenuated by daily intravenous treatment of primates with a mAb directed against intercellular adhesion molecule-1 (ICAM-1) (18). These results are intriguing, not only as proof that leukocyte migration is an important factor in these disease states, but as evidence that new therapies can be based on blocking specific adhesion pathways (17). In this report, we investigate two different strategies of preventing B16-F10 melanoma metastasis by interfering with the adhesion of melanoma cells to Lu-ECAM-1. The first strategy relies on the blocking of murine Lu-ECAM-1 by a monospecific $\mathrm{mAb}$ (6D3). In the second strategy, mice are actively immunized against bovine Lu-ECAM- 1 and, after developing satisfactory antibody titers, are challenged with high lung-metastatic B16-F10 melanoma cells. Both of these "antiadhesion" therapies drastically reduce experimental B16 metastases.

\section{Methods}

Cell culture. Endothelial cells are routinely isolated from thoracic aortas of 18-mo-old bovines, essentially as described by Booyse et al. (19). BAEC are grown in DME medium supplemented with $10 \%$ heat inactivated fetal bovine serum (FBS). After verifying the endothelial cell origin of the cultures with monospecific antibodies against Factor VIII 
antigen, BAEC are frozen in liquid nitrogen at passage 2 or 3 . BAEC used in the outlined experiments are at passage 3 to 10 .

Murine B16 melanoma cell variants (B16-F0 and B16-F10) have been obtained from Dr. I. J. Fidler (University of Texas System Cancer Center, MD Anderson Hospital and Tumor Institute, Houston, TX). B16-F0 are low lung-metastatic and B16-F10 are selected in vivo for high lung colonization (20). Liver-metastatic B16-L8-F10 melanoma cells (21) have been obtained from Dr. D. Rusciano (Friedrich Miescher-Institut, Basel, Switzerland) and KLN205 murine squamous carcinoma cells from the American Type Culture Collection (Rockville, MD). B16 melanoma cells are maintained in Rosewell Park Memorial Institute 1640 (RPMI 1640) medium and KLN205 in MEM, each supplemented with $10 \%$ heat-inactivated FBS.

Modulation of BAEC with lung matrix extracts. BAEC are modulated to express lung-specific endothelial cell adhesion molecules by growing them on extracellular matrix extracts of the lungs $(6,7)$. Extracellular matrix is prepared from the lungs of 18 -mo-old bovines, as described in detail elsewhere $(7,22)$. Lung matrix free of lipids and nucleic acids is extracted overnight at $4^{\circ} \mathrm{C}$ with $0.5 \mathrm{~N}$ acetic acid $(10$ $\mathrm{ml} / \mathrm{g}$ of matrix powder). Extracts are collected by centrifugation $(23,000 \mathrm{~g}$ for $30 \mathrm{~min})$, lyophilized, and stored at $-70^{\circ} \mathrm{C}$. Tissue culture grade plastic ware is coated with lung matrix extracts ( $25 \mu \mathrm{g}$ protein/ $\mathrm{cm}^{2}$ growth surface), solubilized in PBS, pH 7.4. Dishes are incubated overnight at $4^{\circ} \mathrm{C}$, then washed thoroughly with PBS and used for seeding with BAEC $\left(5 \times 10^{4} \mathrm{cells} / \mathrm{cm}^{2}\right.$ growth surface in DME containing $10 \%$ FBS). Unless stated otherwise, BAEC used in the present experiments are grown continuously for $8 \mathrm{~d}$ on lung matrix extracts with transfer of cells to fresh lung matrix-coated dishes every other day.

Monoclonal antibodies. Anti-Lu-ECAM-1 mAb 6D3 $\left(\operatorname{IgG}_{22}\right)$ is produced in Balb/c mice and purified from ascites fluid or hybridoma supernatant on rProtein $\mathrm{A}^{\mathrm{TM}}$ chromatographic matrix (Repligen Corp., Cambridge, MA) as described in detail elsewhere $(15,23)$. MAb 6D3 monospecifically recognizes bovine and murine Lu-ECAM-1 expressed by endothelia of bovine lung postcapillary venules, mouse subpleural and peribronchial venules, and segments of mouse pulmonary veins $(6,15)$. Control mAbs are directed against Trichinella spiralis (6D8; same immunoglobulin class as anti-Lu-ECAM-1 mAb 6D3) (23) and determinants other than Lu-ECAM-1 expressed on the surface of lung matrix-modulated BAEC or BAEC grown on plastic $\left(3 \mathrm{C} 6, \mathrm{IgG}_{1}\right.$; $\left.5 \mathrm{E} 12, \operatorname{lgG}_{1}\right)(15)$.

Immunoaffinity purification of $L u-E C A M-1$. Lu-ECAM-1 is isolated and purified by immunoaffinity chromatography from both detergent extracts of lung matrix-modulated BAEC and murine lungs, using anti-Lu-ECAM-1 mAb 6D3 as described in detail by our laboratory (6). Since the amounts of Lu-ECAM-1 harvested from mouse lungs is insufficient for performing large scale tumor cell-binding studies, and since murine and bovine Lu-ECAM-1 express similar binding affinities for B16 melanoma cells, all tumor cell-binding experiments are conducted with bovine Lu-ECAM-1. The purity of the Lu-ECAM1 preparations used in the present study is assessed after electrophoresis by silver staining of SDS polyacrylamide gels. Only preparations that yield a single protein band of $90 \mathrm{kD}$ are used here.

Tumor cell adhesion assay. Wells of 96-well plates are coated with various concentrations of immunoaffinity purified Lu-ECAM-1 (10$200 \mathrm{ng} / \mathrm{ml}$ ) or $0.4 \%$ BSA in PBS, pH 7.4, 0.1\% dodecylpoly(ethyleneglycoether), (Thesit) overnight at $4^{\circ} \mathrm{C}$. Wells are washed three times with RPMI 1640 , seeded with $2 \times 10^{4}$ tumor cells previously labeled with [ $\left.{ }^{3} \mathrm{H}\right]$ thymidine $(2 \mathrm{Ci} / \mathrm{mmol} ; 200 \mu \mathrm{Ci} / \mathrm{ml}$ in RPMI 1640 for $24 \mathrm{~h}$ at $37^{\circ} \mathrm{C}$ ), then spun onto the coated wells at $15 \mathrm{~g}$ for $1 \mathrm{~min}(7)$. After an incubation period of $10 \mathrm{~min}$ at $37^{\circ} \mathrm{C}$, nonadherent tumor cells are spun off at $150 \mathrm{~g}$ for $5 \mathrm{~min}$. Adherent tumor cells are dissolved in 1\% SDS and counted in a liquid scintillation counter. Tumor cell attachment is determined as the percent cells bound of the total cells seeded ([percentage counts per minute tumor cells bound to Lu-ECAM-1]-[percentage counts per minute tumor cell bound to BSA]) and is expressed as a function of the concentration of Lu-ECAM- 1 in the coating solution as well as the number of Lu-ECAM-1 molecules $/ \mu \mathrm{m}^{2}$ of plastic surface, the latter being determined from the counts per minute of plastic-ad- herent ${ }^{125}$ I-labeled Lu-ECAM-1. Inhibition of tumor cell adhesion to Lu-ECAM-1 is determined after having incubated Lu-ECAM-1 coated wells with different mAbs (6D3, 6D8, 3C6; each $10 \mu \mathrm{g} / \mathrm{ml}$ ) for $1 \mathrm{~h}$ at room temperature or in the presence of various concentrations of soluble Lu-ECAM-1. Neutrophils and T lymphocytes prepared from bovine peripheral blood $(24,25)$ are tested in a similar manner for adhesion to Lu-ECAM-1.

To determine the effect of divalent cations on tumor cell binding, Lu-ECAM-1-coated wells are washed once with $\mathrm{Ca}^{2+}$ - and $\mathrm{Mg}^{2+}$-free HBSS containing $5 \mathrm{mM}$ EDTA, followed by three washes of $\mathrm{Ca}^{2+}$ - and $\mathrm{Mg}^{2+}$-free HBSS (26). $\mathrm{CaCl}_{2}$ and $\mathrm{MgCl}_{2}$ are then added to $\mathrm{HBSS}$ at the indicated concentrations. The Lu-ECAM-1-coated dishes are preequilibrated for $15 \mathrm{~min}$ in the supplemented buffer, and adhesion assays performed as described above.

Inhibition of lung colonization by anti-Lu-ECAM-1 $m A b$. Anti-LuECAM-1 mAb 6D3 is tested for its efficacy in inhibiting the formation of experimental lung metastases in a standard lung colony assay (20). Syngeneic mice (seven to eight mice per group) are inoculated with anti-Lu-ECAM-1 mAb 6D3 (200 $\mu \mathrm{g} / 200 \mu \mathrm{l} \mathrm{PBS/mouse)} \mathrm{via} \mathrm{the} \mathrm{lateral}$ tail vein, followed 1,6 , or $24 \mathrm{~h}$ later with an intravenous injection of $10^{5}$ tumor cells/200 $\mu \mathrm{l}$ PBS/mouse (B16-F0, B16-F10, B16-L8-F10, or $\mathrm{KLN} 205$ ). Control animals receive mAbs $6 \mathrm{D} 8,3 \mathrm{C6}$, or $5 \mathrm{E} 12$ (15), or PBS alone. Mice are killed $21 \mathrm{~d}$ after tumor cell inoculation. The effect of the treatment is determined from the number of lung colonies formed in experimental and control mice (median and range). Statistical analyses are by Student's $t$ test.

Immunization of mice with $\mathrm{Lu}$-ECAM-1. Immunoaffinity-purified Lu-ECAM-1 is prepared from detergent extracts of lung matrix-modulated BAEC. Syngeneic female mice, 6-8 wk old, are immunized by injection of $10 \mu \mathrm{g}$ s.c. of Lu-ECAM-1 emulsified in $250 \mu \mathrm{l}$ of complete Freund's adjuvant. Mice are reinoculated 4 wk later with the same antigen concentration emulsified in incomplete Freund's adjuvant. Sera from immunized mice are probed for anti-Lu-ECAM-1 titers $6 \mathrm{wk}$ after the initial immunization, using an ELISA method (23). Mice with acceptable antibody titers $(\geq 1: 200)$ are inoculated intravenously with high lung-metastatic B16-F10 melanoma cells or KLN205 squamous carcinoma cells ( $10^{5}$ tumor cells/mouse). Mice immunized with saline expressing no anti-Lu-ECAM-1 antibody titers serve as controls. Mice are killed $21 \mathrm{~d}$ after tumor cell inoculation and the number of lung colonies counted (20). A complete autopsy is performed to assess potential toxic effects of the immunization. No toxic effects are observed in these short term experiments.

Lung clearance rates of ${ }^{125}$ I-5-Iodo-2'-deoxyuridine-labeled B16F10 melanoma cells. C57Bl/6 mice (male; 6 wk old) are injected via the lateral tail vein with anti-Lu-ECAM-1 mAb 6D3 $(200 \mu \mathrm{g} / 200 \mu \mathrm{l})$ and, 1 h later, with $10^{5}$ of ${ }^{125} \mathrm{I}$-5-iodo-2'-deoxyuridine ( ${ }^{125}$ IUDR; sp act: 2,200 $\mathrm{Ci} / \mathrm{mmol} ; 1 \mu \mathrm{Ci} / \mathrm{ml}$ of medium; $24 \mathrm{~h}$ ) labeled B16-F10 melanoma cells. Mice are sacrificed by cervical dislocation at various times after B16F10 inoculation [ $1 \mathrm{~min} ; 30 \mathrm{~min} ; 1 \mathrm{~h} ; 3 \mathrm{~h} ; 6 \mathrm{~h} ; 12 \mathrm{~h} ; 1 \mathrm{~d} ; 2 \mathrm{~d} ; 3 \mathrm{~d} ; 5 \mathrm{~d}$ (3 mice per time point)] and exsanguinated. Organs are removed immediately, placed in individual vials containing $70 \%$ ethanol, and processed for gamma-counting, as described by Fidler (27). The number of viable tumor cells in each organ is determined from the radioactivity counts, by using the ratio of counts per minute to cells in the original inoculum. Untreated mice serve as controls.

\section{Results}

Melanoma cell-binding Lu-ECAM-1. Bovine Lu-ECAM-1 immobilized on protein-binding polystyrene surfaces binds high lung-metastatic B16-F10 melanoma cells in significantly larger numbers than their intermediate and low lung-metastatic counterparts B16-L8-F10 and B16-F0, respectively (Fig. 1). Maximal binding is observed at a density of $\sim 2.4 \times 10^{2} \mathrm{Lu}-$ ECAM-1 molecules per $\mu \mathrm{m}^{2}$ of plastic surface (100 $\mathrm{ng}$ of LuECAM- $1 / \mathrm{ml}$ of coating solution). A slight decrease in the bind- 

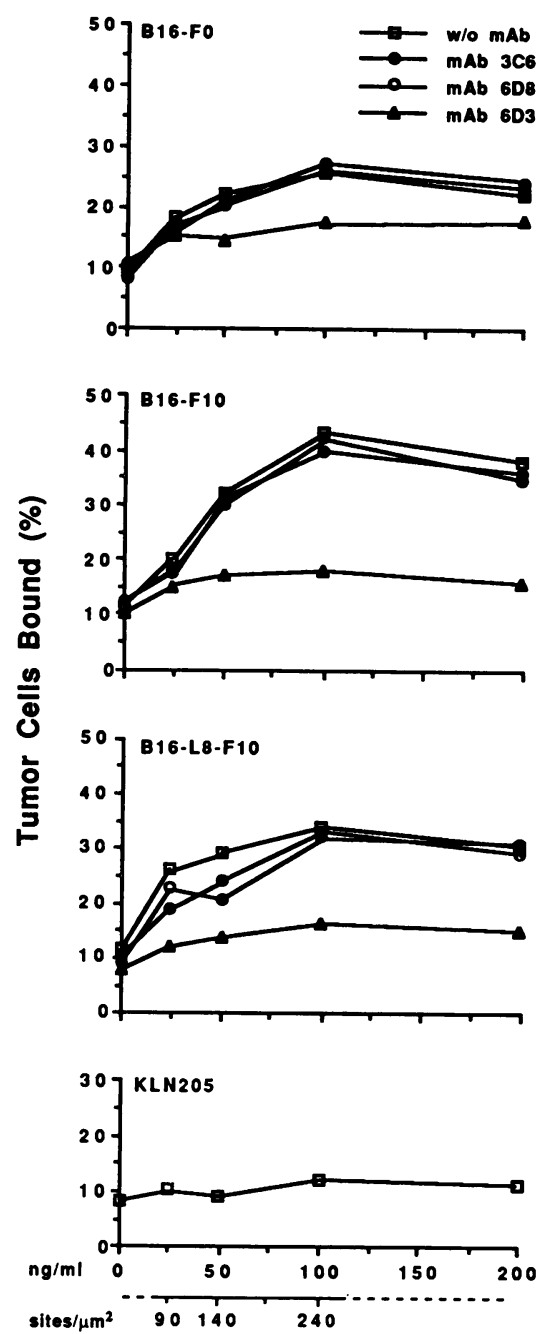

LU-ECAM-1
Figure 1. Lu-ECAM-1 binding and binding inhibition curves for B16 melanoma cell variants. Tumor cell attachment is determined as the percent cells bound of the total number of cells seeded ([percent counts per minute tumor cells bound to Lu-ECAM-1][percent counts per minute tumor cells bound to $0.4 \% \mathrm{BSA}]$ ) and expressed as a function of the Lu-ECAM-1 coating concentration (nanogram/milliliter) and numerical density (sites/micrometer ${ }^{2}$ of plastic surface) (open squares). Adhesion inhibition experiments are performed in the presence of different mAbs each used at a concentration of $10 \mu \mathrm{g} /$ $\mathrm{ml}$. Data are the means of three experiments, each with triplicate determinations (SDs are within $10 \%$ of the means).

ing of B16 melanoma cells to plastic wells coated at a higher concentration than $100 \mathrm{ng}$ of $\mathrm{Lu}-\mathrm{ECAM}-1 / \mathrm{ml}$ is paralleled by a decrease in the number of Lu-ECAM- 1 molecules $/ \mu \mathrm{m}^{2}$ of plastic surface from $2.4 \times 10^{2}$ at $100 \mathrm{ng}$ to $2.1 \times 10^{2}$ at $200 \mathrm{ng}$ of Lu-ECAM- $1 / \mathrm{ml}$ of coating solution. Binding of B16 melanoma cells is dependent upon the presence of $\mathrm{Ca}^{2+}$ in HBSS adhesion medium (1 mM), but is unaffected by $\mathrm{Mg}^{2+}$ (Fig. 2). The binding affinity of B16 cell variants for Lu-ECAM- 1 correlates well with their propensity of colonizing the lungs (Table I). B16-F10 bind in large numbers to Lu-ECAM-1 and produce numerous experimental metastases, while B16-L8-F10 express intermediate and B16-F0-low Lu-ECAM-1 adhesion and, correspondingly, metastatic colonization. Lu-ECAM-1 appears to be a melanoma-specific endothelial cell adhesion molecule that fails to bind other lung-metastatic tumor cell lines (e.g., murine KLN205 squamous carcinoma cells), as well as lymphocytes and neutrophils isolated from bovine peripheral blood (Figs. 1 and 3).

The preferential binding of lung-metastatic B16 melanoma cells to Lu-ECAM-1 is significantly inhibited by anti-LuECAM-1 mAb 6D3 (Fig. 1). At each coating density of LuECAM-1, mAb $6 \mathrm{D} 3(10 \mu \mathrm{g} / \mathrm{ml})$ reduces binding of the three

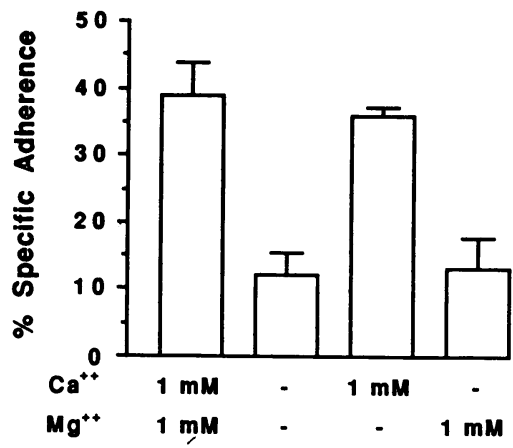

Figure 2. B16-F10 binding to Lu-ECAM-1 in the presence of divalent cations. Lu-ECAM1 coated wells $(100 \mathrm{ng} /$ $\mathrm{ml}$ ) washed once with $\mathrm{Ca}^{2+}$ - and $\mathrm{Mg}^{2+}$-free HBSS containing $5 \mathrm{mM}$ EDTA and three times with $\mathrm{Ca}^{2+}$ - and $\mathrm{Mg}^{2+}$. free HBSS, are preequilibrated with HBSS containing the indicated concentration of $\mathrm{CaCl}_{2}$ and $\mathrm{MgCl}_{2}$. An adhesion assay is then performed in the same HBSS as described in the text. Data represent the means and SDs from duplicate experiments, each with triplicate determinations.

B16 melanoma cell variants to slightly lower levels than the low lung-metastatic B16-F0 is able to bind to Lu-ECAM-1. Control mAbs 3C6 and 6D8 have no effect on the binding of B16 melanoma cell variants to Lu-ECAM-1. Binding of lung-metastatic B16 melanoma cells to Lu-ECAM-1 coated dishes is also competitively inhibited in a dose-dependent manner by soluble LuECAM-1 supplemented in the assay medium (Fig. 4). Maximal inhibition is achieved at the same Lu-ECAM-1 concentration that has been used for the coating of the plastic dishes, namely $100 \mathrm{ng} / \mathrm{ml}$. Higher Lu-ECAM-1 concentration have no further inhibitory effect (data not shown).

Inhibition of melanoma lung colonization by anti-LuECAM-1 mAb 6D3. Anti-Lu-ECAM-1 mAb 6D3 shown to efficiently inhibit the binding of lung-metastatic B16 melanoma cells to Lu-ECAM-1 in vitro is tested for its efficacy to prevent experimental lung metastases in a standard in vivo lung colony assay. Mice passively immunized with anti-Lu-ECAM-1 mAb 6D3 $1 \mathrm{~h}$ before an intravenous challenge with B16-F10 cells exhibit a more than $90 \%$ reduction in the number of lung colonies when compared with mice that have received control $\mathrm{mAbs}$ of the same immunoglobulin isotype (6D8), or against endothelial cell surface determinants other than Lu-ECAM-1 (3C6 and 5E12) (Table I). The antimetastatic effect of $\mathrm{mAb}$ 6D3 is still noticeable, if B16-F10 are injected 6 or $24 \mathrm{~h}$ after treatment of mice with mAb 6D3, although inhibition of metastasis is now only $41 \%$ and $33 \%$, respectively. As expected, anti-Lu-ECAM-1 mAb 6D3 also efficiently inhibits the colonization of the lungs by B16-L8-F10 (an intermediate lung-metastatic and moderate liver-metastatic B16 melanoma variant), but has no inhibitory effect on the propensity of this cell line to colonize the liver (Table I). However, mAb 6D3 has no effect on the metastatic performance of other lung-metastatic tumor cell types (e.g., KLN205 squamous carcinoma cells). These findings suggest that different tumor cell types recognize distinct Lu-ECAMs and that inhibition of/ung colonization by B16-F10 is not mediated by nonspecific, steric blocking of adhesion, as indicated by the inability of control mAbs $3 \mathrm{C} 6$ and $5 \mathrm{E} 12$, both detecting endothelial cell surface determinants other than Lu-ECAM-1, to inhibit lung colonization of B16 melanoma cells.

To further substantiate that the interruption of the metastatic cascade occurs at the site of tumor cell adhesion to lung endothelium, we have compared lung clearance rates of tail 
Table I. Effect of Anti-Lu-ECAM-1 mAb $6 D 3$ on Experimental Metastases of B16 Melanoma Cells

\begin{tabular}{|c|c|c|c|c|c|c|}
\hline \multirow[b]{2}{*}{ Tumor cells } & \multirow[b]{2}{*}{ Number of mice } & \multirow[b]{2}{*}{$\mathrm{mAb}$} & \multirow[b]{2}{*}{ Time* } & \multicolumn{3}{|c|}{ Number of tumor colonies (Median [Range]) } \\
\hline & & & & Lung & Liver & $P$ (lung) \\
\hline & & & $h$ & & & \\
\hline B16-F0 & 7 & - & - & $5(0-25)$ & 0 & - \\
\hline B16-F10 & 7 & - & - & $180(145->200)$ & 0 & - \\
\hline B16-F10 & 7 & 6D8 & 1 & $171(69->200)$ & 0 & 0.74 \\
\hline B16-F10 & 7 & $3 \mathrm{C} 6$ & 1 & $158(98->200)$ & 0 & 0.93 \\
\hline B16-F10 & 7 & $5 \mathrm{E} 12$ & 1 & $174(125->200)$ & 0 & 0.71 \\
\hline B16-F10 & 7 & 6D3 & 1 & $15(7-42)$ & 0 & $<0.0001$ \\
\hline B16-F10 & 7 & 6D3 & 6 & $107(42-134)$ & 0 & $<0.001$ \\
\hline B16-F10 & 7 & 6D3 & 24 & $121(78-167)$ & 0 & $<0.01$ \\
\hline B16-L8-F10 & 8 & - & - & $92(58-135)$ & $6(1-17)$ & - \\
\hline B16-L8-F10 & 8 & 6D8 & 1 & $79(47-113)$ & $6(1-15)$ & 0.36 \\
\hline B16-L8-F10 & 8 & 6D3 & 1 & $22(9-30)$ & $5(1-11)$ & $<0.001$ \\
\hline KLN205 & 7 & - & - & $182(118->200)$ & 0 & - \\
\hline KLN205 & 7 & 6D3 & 1 & $178(117->200)$ & 0 & 0.40 \\
\hline
\end{tabular}

* Time in hours between mAb administration and challenge with tumor cells. vein-injected B16-F10 melanoma cells in untreated C57Bl/6 mice and in mice treated with anti-Lu-ECAM-1 mAb 6D3. Lung clearance is rapid in both animal groups and is accomplished primarily within the first $12 \mathrm{~h}$ after intravenous injection of B16-F10 (Table II). At the end of this period only $2.11 \%$ of the initial tumor cell inoculum remains in the lungs of untreated mice, compared with $1.32 \%$ in mice treated with antiLu-ECAM-1 mAb 6D3. The more rapid tumor cell clearance from the lungs of anti-Lu-ECAM-1 mAb 6D3 pretreated mice is paralleled by an expected increase in the number of tumor cells in liver, spleen, and kidney relative to untreated controls. By 3 to $5 \mathrm{~d}$ after intravenous inoculation of tumor cells, the number of lung-bound tumor cells has further decreased to correspond approximately with the number of lung colonies counted 3 wk later in each of the animal groups (Tables I and II). The remaining organs are all cleared from unspecifically arrested tumor cells within 3 to $5 \mathrm{~d}$ after tail vein injection of
B16-F10 melanoma cells, although a slightly slower pace of clearance is observed in the treated animal group, as illustrated by the clearance rates of B16-F10 from livers and kidneys (Table II).

Inhibition of melanoma lung colonization by active immunization against Lu-ECAM-1. Active immunization of mice against bovine Lu-ECAM-1 purified by immunoaffinity chromatography from detergent extracts of superconfluent lung matrix-modulated BAEC also prevents metastatic colonization of the lungs by B16-F10 melanomas (Table III; Fig. 5). The efficiency of inhibition of experimental metastases is dependent upon the anti-Lu-ECAM-1 serum titer of the immunized mice. At a titer of 1:1,000 an average of $21 \pm 6$ B16-F10 tumor colonies are counted, but only $9 \pm 3$ colonies are observed in mice with serum titers of $1: 2,000(P<0.05)$ (Table III). In contrast, mice immunized with saline alone, having no detectable anti-Lu-ECAM-1 serum titers, exhibit over 200 B16-F10

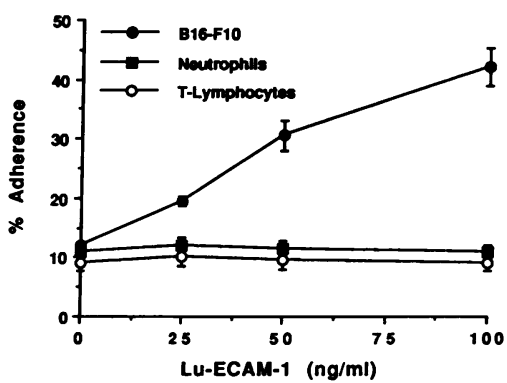

ECAM-1, and an adhesion assay is performed as described in the text; except, cells adhering to Lu-ECAM-1-coated dishes are released with trypsin and counted in a hemocytometer. Data represent the means and SDs of two experiments, each with triplicate determinations.

Figure 3. Binding of B16-F10 melanoma cells and peripheral blood neutrophils and T lymphocytes to LuECAM-1. Murine B16F10 melanoma cells and bovine blood neutrophils and $\mathrm{T}$ lymphocytes are seeded onto cytes are seeded onto microtiter wells coated th

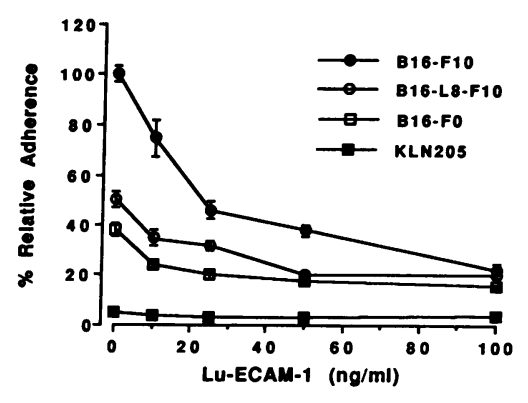

the indicated concentrations of soluble Lu-EC concent ane same fortion of soluble Lu-ECAM-1. Data represent means and SDs from three experiments, each with triplicate determinations. 
Table II. Organ Clearance of ${ }^{125}$ IUDR-labeled B16-F10 Melanoma Cells Injected Intravenously into C57Bl/6 Mice

\begin{tabular}{|c|c|c|c|c|c|c|}
\hline \multirow{3}{*}{$\begin{array}{l}\text { Time after } \\
\text { tumor cell } \\
\text { injection }\end{array}$} & \multicolumn{6}{|c|}{ Number of tumor cells* } \\
\hline & \multicolumn{2}{|c|}{ Lung } & \multicolumn{2}{|c|}{ Liver } & \multicolumn{2}{|c|}{ Kidney } \\
\hline & $-6 \mathrm{D} 3$ & $+6 \mathrm{D} 3^{\ddagger}$ & $-6 \mathrm{D} 3$ & $+6 \mathrm{D} 3^{\ddagger}$ & $-6 \mathrm{D} 3$ & $+6 \mathrm{D}^{\ddagger}$ \\
\hline $1 \mathrm{~min}$ & 49,580 & 48,700 & 1,270 & 1,460 & 253 & 285 \\
\hline $30 \mathrm{~min}$ & 36,970 & 24,260 & 4,060 & 6,310 & 1,390 & 1,910 \\
\hline $1 \mathrm{~h}$ & 23,220 & 16,900 & 2,800 & 4,880 & 600 & 1,150 \\
\hline $3 \mathrm{~h}$ & 7,560 & 4,280 & 940 & 1,250 & 400 & 1,070 \\
\hline $6 \mathrm{~h}$ & 2,500 & 1,550 & 600 & 1,150 & 380 & 1,020 \\
\hline $12 \mathrm{~h}$ & 2,110 & 1,320 & 480 & 1,080 & 370 & 970 \\
\hline $24 \mathrm{~h}$ & 1,310 & 610 & 440 & 1,010 & 260 & 880 \\
\hline $48 \mathrm{~h}$ & 456 & 184 & 230 & 413 & 124 & 370 \\
\hline $72 \mathrm{~h}$ & 287 & 72 & 90 & 118 & 28 & 90 \\
\hline $120 \mathrm{~h}$ & 214 & 28 & 0 & 0 & 0 & 0 \\
\hline
\end{tabular}

* Mean number of tumor cells per organ (four mice per time point); $\mathrm{SD}<15 \%$ of mean. ${ }^{\ddagger}$ Anti-Lu-ECAM-1 mAb 6D3 $(200 \mu \mathrm{g} / 200 \mu \mathrm{l}$ PBS) was injected $1 \mathrm{~h}$ before the intravenous injection of $10^{5} \mathrm{~B} 16-\mathrm{F} 10$ melanoma cells.

melanoma lung colonies. Similar to passive immunization, active immunization against Lu-ECAM-1 has no effect on the metastatic performance of KLN205.

\section{Discussion}

The melanoma cell-binding endothelial cell adhesion molecule Lu-ECAM-1 is localized by immunohistochemical techniques in lung blood vessels, where it occurs predominantly on the endothelial cell lumenal membrane of subpleural venules, and, to a lesser extent, other pulmonary venules and veins, but not in endothelia from other tissues (6). This tissue- and vessel caliber-dependent distribution of Lu-ECAM-1 correlates precisely with the distribution of experimental B16 melanoma metastases in the lungs of syngeneic $\mathrm{C} 57 \mathrm{Bl} / 6$ mice. The $90-\mathrm{kD}$ Lu-ECAM-1 is part of the constitutive vascular phenotype of mouse lungs, and, thus, is distinctly different from the cytokine-inducible endothelial cell adhesion molecules such as endothelial/leukocyte adhesion molecule-1 (ELAM-1) and inducible cell adhesion molecule-110 (INCAM-110) (28) that, in addition to serving as adhesion molecules for leukocytes, also

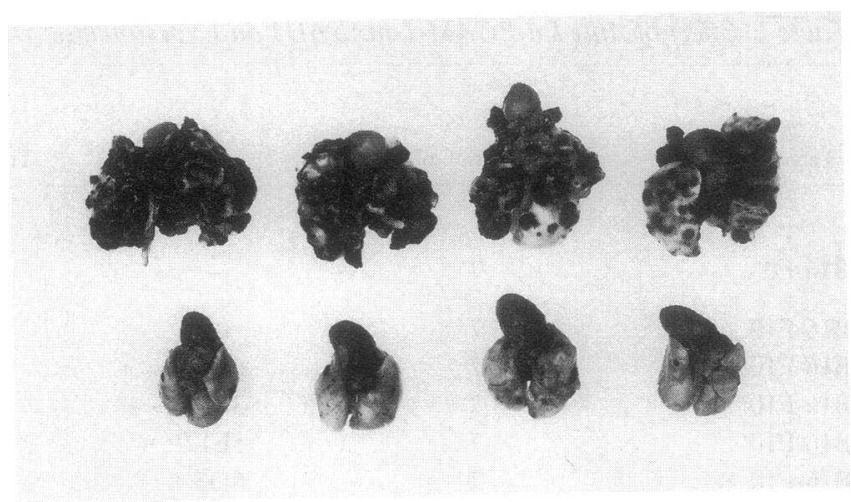

Figure 5. Inhibition of B16-F10 experimental lung metastases by active immunization with Lu-ECAM-1. (Row 1) Numerous lung colonies are present in saline-immunized mice; (Row 2) Only a few colonies are observed in mice immunized against Lu-ECAM-1.

serve the adhesion of distinct tumor types. In accordance, LuECAM-1 is not upregulated by any of the tested cytokines (e.g., TNF- $\alpha$, LPS, rIL- $1 \alpha$ ) that have been successfully employed in regulating the expression of ELAM-1 and INCAM-110 (6). LuECAM-1 is also not upregulated by acute inflammatory processes and does not bind to neutrophils and lymphocytes from peripheral bovine blood. Its nonreducibility with $\beta$-mercaptoethanol indicates that it does not belong to the integrin family of adhesion molecules (29). This finding is consistent with the inability of Lu-ECAM-1 to react with anti-integrin $\beta_{1}$ and $\beta_{3}$ chain antibodies $(30,31)$. Thus, Lu-ECAM-1 is the first constitutively expressed endothelial cell adhesion molecule involved in selectively mediating the preferred metastasis of B16F10 to the lungs.

The dependence of cancer cells on endothelial cell adhesion molecules in metastasis formation has been exploited in the present study to develop an antiadhesion therapy for B16 melanoma metastasis. The idea of interfering with tumor cell adhesion to host cells or stroma to interrupt the metastatic cascade, and, thus, the formation of secondary tumor colonies, is by no means a novel one. Most notable examples of experimental antiadhesion therapies in cancer metastasis are the blocking of tumor cell-induced platelet aggregation and the inhibition of tumor cell binding to the matrix macromolecules laminin and fibronectin. In the first group of experiments metastasis is inhibited by blocking tumor cell adhesion to GPIIb/IIIa integrin or

Table III. Inhibition of B16-F10 Lung Colony Formation by Active Immunization with Lu-ECAM-1

\begin{tabular}{|c|c|c|c|c|c|}
\hline \multirow[b]{2}{*}{ Tumor cells } & \multirow[b]{2}{*}{ No. of mice } & \multirow[b]{2}{*}{ Antigen } & \multirow[b]{2}{*}{ Anti-Lu-ECAM-1 titer } & \multicolumn{2}{|c|}{ No. of lung colonies } \\
\hline & & & & Per mouse & Group mean \pm SD \\
\hline B16-F10 & 3 & Lu-ECAM-1 & $1: 1,000$ & $16,21,28$ & $21 \pm 6^{*}$ \\
\hline $\mathrm{B} 16-\mathrm{F} 10$ & 3 & Lu-ECAM-1 & $1: 2,000$ & $7,9,12$ & $9 \pm 3^{*}$ \\
\hline B16-F10 & 6 & Saline & 0 & $\begin{array}{l}>200,>200,>200 \\
>200,>200,>200\end{array}$ & $>200$ \\
\hline KLN205 & 6 & Lu-ECAM-1 & $\begin{array}{l}1: 200 \text { to } \\
1: 1,000\end{array}$ & $32,54,76,98,146,157$ & $94 \pm 49$ \\
\hline KLN205 & 6 & Saline & 0 & $54,76,92,112,126,145$ & $101 \pm 33$ \\
\hline
\end{tabular}

${ }^{*} 0.01<P<0.05$ (B16-F10: group 1:1,000 vs group 1:2,000). 
GMP-140 selectin with various lactose derivates, including methyl- $\beta$-lactoside and lactosylceramide $(32,33)$. In the second group of experiments the inhibition of metastasis is achieved by mAbs or synthetic peptides that block the binding sites of tumor cells for laminin or fibronectin. An mAb directed against laminin-binding protein expressed on the surface of B16 melanoma cells has been shown to significantly inhibit the number of experimental metastases (34). Similarly, synthetic peptides that inhibited the binding of tumor cells to fibronectin (GRGDS) $(35,36)$ and laminin (YIGSR) (37) are able to block tumor cell adhesion to these matrix macromolecules in vitro, and, in accordance, reduce the number of experimental metastases in vivo (37-39).

In this report, we show that inhibition of tumor cell binding to endothelia of the secondary target organ results in a significant reduction of experimental metastases. This inhibition can be achieved by two antiadhesion strategies. The first relies on the inoculation of anti-Lu-ECAM-1 mAb 6D3 before challenge of the host animal with B16 melanoma cells. The second is based on an active immunization of tumor-recipient mice with immunoaffinity-purified Lu-ECAM-1. These experiments show that lung metastasis of B16-F10 melanoma cells critically depends upon the adherence to Lu-ECAM-1, and that other lung-metastatic tumor cells that do not adhere to Lu-ECAM-1 in vitro are not affected in their metastatic performance. The importance of Lu-ECAM-1 in the formation of lung metastases is further underscored by the clearance rates of intravenous-inoculated B16-F10 melanoma cells from the lungs of untreated mice and mice treated with anti-Lu-ECAM$1 \mathrm{mAb} 6 \mathrm{D} 3$. These rates show that $\mathrm{B} 16-\mathrm{F} 10$ are more rapidly cleared from anti-Lu-ECAM-1 mAb 6D3-treated mice than from untreated controls, and that $\mathrm{mAb} 6 \mathrm{D} 3$ is effective during the early phase of metastatic lung colonization, i.e., during the phase that precedes tumor cell extravasation $(0-48 \mathrm{~h})$ and which is dominated by tumor cell adhesion to endothelium (40). Our studies and those previously reported on the inhibition of tumor cell binding to platelets and matrix macromolecules (predominantly basement membrane components) show that the metastatic cascade can be successfully interrupted at various stages, resulting in reduction of metastases $(38,39)$. The interruption of the metastatic cascade at the endothelial level has the distinct advantage that cancer cells are kept within the circulation where they continue to be subjected to elimination by hemodynamic and geometric forces shown to be most efficient in their eradication $(41,42)$.

Our results on the inhibition of metastases by antiadhesion therapies complement recent reports on the successful use of antiadhesion therapies in the combat of excessive leukocyte participation and associated tissue damage in autoimmune, allergic, and inflammatory disorders (16-18). Such antiadhesion therapies have been tested in a number of animal models of inflammation using mAbs to various adhesion proteins. Most notably, these antibodies have been directed against CD18, and, in some instances, CD11b and CD54 (16). The results have been striking and have promoted interest in clinical trials in myocardial infarction, shock, and renal allografts rejection. In cancer, our experiments show that antiadhesion therapies directed at interfering with the adhesion between blood-borne cancer cells and organ-specific vascular endothelia could be exploited in preventing the formation of secondary tumor colonies in distant organs. However, the applicability of such therapies is somewhat restrained by the fact that various cancer types appear to recognize and adhere to distinct endothelial cell adhesion molecules $(1-8,43,44)$. This is not only emphasized by the selective binding of B16 melanoma cells to Lu-ECAM-1 in lung colony formation (6), but also by more recent findings in our laboratory that rat mammary carcinoma cells metastasize to the lungs by recognizing and adhering to a distinctly different endothelial glycoprotein than Lu-ECAM-1 (Johnson, R. C., and B. U. Pauli, manuscript in preparation), and by the transference of metastatic potential to nonmetastatic BSP73AS pancreatic carcinoma cells after transfection with p-Meta-1 cDNA, encoding a variant of CD44 that binds to yet another endothelial cell adhesion molecule (45). Whether various groups of cancer cells (e.g., carcinomas) recognize a common endothelial cell adhesion molecule for metastatis to a specific organ such as the lungs or the liver is currently under investigation. If this is the case, an antiadhesion therapy of metastases will obviously become much more appealing and clinically useful.

\section{Acknowledgments}

The authors wish to thank Ursula Nanna, Joanne Widom, and Marwan El-Sabban for their expert technical assistance.

This work was supported by U. S. Public Health Service grant CA47668 from the National Cancer Institute, and a grant from the Cornell Biotechnology Program.

\section{References}

1. Alby, L., and R. Auerbach. 1984. Differential adhesion of tumor cells to capillary endothelial cells in vitro. Proc. Natl. Acad. Sci. USA. 81:5739-5743.

2. Roos, E., A. Tulp, O. P. Middelkoop, and I. V. van de Pavert. 1984. Interactions between lymphoid tumor cells and isolated liver endothelial cells. $J$. Natl. Cancer Inst. 72:1173-1180.

3. Auerbach, R., W. C. Lu, E. Pardon, F. Gumkowski, G. Kaminska, and M. Kaminski. 1987. Specificity of adhesion between murine tumor cells and capillary endothelium: an in vitro correlate of preferential metastasis in vivo. Cancer Res. 47:1492-1496.

4. Bargatze, R. F., N. W. Wu, I. L. Weissman, and E. C. Butcher. 1987. High endothelial venule binding as a predictor of the dissemination of passaged murine lymphomas. J. Exp. Med. 166:1125-1131.

5. Nicolson, G. L., P. N. Belloni, R. J. Tressler, K. Dulski, T. Inoue, and P. G. Cavenaugh. 1989. Adhesive, invasive and growth properties of selected metastatic variants of murine large-cell lymphoma. Invasion \& Metastasis. 9:102-116.

6. Zhu, D., C. F. Cheng, and B. U. Pauli. 1991. Mediation of lung metastasis of murine melanomas by a lung-specific endothelial cell adhesion molecule. Proc. Natl. Acad. Sci. USA. 88:9568-9572.

7. Pauli, B. U., and C. L. Lee. 1988. Organ preference of metastasis: the role of organ-specifically modulated endothelial cells. Lab. Invest. 58:379-387.

8. Pauli, B. U., H. G. Augustin-Voss, M. E. El-Sabban, R. C. Johnson, and D. A. Hammer. 1990. Organ-preference of metastasis: the role of endothelial cell adhesion molecules. Cancer Metastasis Rev. 9:175-189.

9. Berg, E. L., L. A. Goldstein, M. A. Jutila, M. Nakache, L. J. Picker, P. R. Streeter, N. W. Wu, D. Zhou, and E. C. Butcher. 1989. Homing receptors and vascular addressins: cell adhesion molecules that direct lymphocyte traffic. Immunol. Rev. 108:5-18.

10. Yednock, T. A., and S. D. Rosen. 1989. Lymphocyte homing. Adv. Im munol. 44:313-378.

11. Springer, T. A. 1990. Adhesion receptors of the immune system. Nature (Lond.). 346:425-434.

12. Paget, S. 1889. The distribution of secondary growths in cancer of the breast. Lancet. i:571-573.

13. Sugerbaker, E. V. 1981. Patterns of metastasis in human malignancies. Cancer Biol. Rev. 2:235-278.

14. Murphy, P., P. Alexander, P. V. Senior, J. Flemming, N. Kirkham, and I. Taylor. 1988. Mechanisms of organ selective tumour growth by blood-borne cancer cells. Br. J. Cancer. 57:19-31.

15. Zhu, D., and B. U. Pauli. 1991. Generation of monoclonal antibodies directed against organ-specific endothelial cell surface determinants. J. Histochem. Cytochem. 39:1137-1142.

16. Carlos, T. M., and J. M. Harlan. 1990. Membrane proteins involved in phagocyte adherence to endothelium. Immunol. Rev. 114:5-28. 
17. Osborn, L. 1990. Leukocyte adhesion to endothelium in inflammation. Cell. 62:3-6.

18. Wegner, C. D., R. H. Gundel, P. Reilly, N. Haynes, L. G. Letts, and R. Rothlein. 1990. Intercellular adhesion molecule-1 (ICAM-1) in the pathogenesis of asthma. Science (Wash. DC). 247:456-459.

19. Booyse, F. M., B. J. Sedlak, and M. E. Rafelson, Jr. 1975. Culture of arterial endothelial cells: characterization and growth of bovine aortic cells. Thromb. Diath. Haemorrh. 34:825-839.

20. Fidler, I. J. 1973. Selection of successive tumour lines for metastasis. Nature (Lond.). 242:148-149.

21. Tao, T., A. Matter, K. Vogel, and M. M. Burger. 1979. Liver colonizing melanoma cells selected from B16 melanoma. Int. J. Cancer. 23:854-857.

22. Rojkind, M., Z. Gatmaitan, S. Mackensen, M. A. Giabrone, P. Ponce, and L. M. Reid. 1980. Connective tissue biomatrix: its isolation and utilization for long-term cultures of normal rat hepatocytes. J. Cell Biol. 87:255-263.

23. Zhu, D., and R. G. Bell. 1990. Trichinella spiralis: murine strain variation in response to monoclonally defined, protective, nonstage-specific antigens. Exp. Parasitol. 70:330-343.

24. Ferrante, A., and Y. H. Thong. 1980. Simultaneous preparation of mononuclear and polymorphonuclear leucocytes from horse blood on Ficoll-Hypaque medium. J. Immunol. Methods. 34:279-285.

25. Korenaga, M., C. H. Wang, R. G. Bell, D. Zhu, and A. Ahmad. 1989. Intestinal immunity to Trichinella spiralis is a property of $0 \times 8^{-} \mathrm{OX} 22^{-} \mathrm{T}$-helper cells that are generated in the intestine. Immunology. 66:588-594.

26. Marlin, S. D., and T. A. Springer. 1987. Purified intercellular adhesion molecule-1 (ICAM-1) is a ligand for lymphocyte function-associated antigen-1 (LFA-1). Cell. 51:813-819.

27. Fidler, I. J. 1970. Metastasis: quantitative analysis of distribution and fate of tumor emboli labeled with ${ }^{125}$ I-5-iodo-2'-deoxyuridine. J. Natl. Cancer Inst. 45:773-782.

28. Rice, G. E., and M. P. Bevilacqua. 1989. An inducible endothelial cell surface glycoprotein mediates melanoma adhesion. Science (Wash.). 246:13031306.

29. Hynes, R. O. 1987. Integrins: a family of cell surface receptors. Cell. 48:549-555.

30. Marcantonio, E. E., and R. O. Hynes. 1988. Antibodies to the conserved cytoplasmic domain of the integrin $\beta 1$ subunit react with proteins in vertebrates, invertebrates, and fungi. J. Cell Biol. 106:1765-1772.

31. Cheresh, D. A., and R. C. Spiro. 1987. Biosynthetic and functional properties of an Arg-Gly-Asp-directed receptor involved in human melanoma cell at- tachment to vitronectin, fibrinogen and von Willebrand factor. J. Biol. Chem. 262:17703-17711.

32. Oguchi, H., T. Toyokuni, B. Dean, H. Ito, E. Otsuji, V. L. Jones, K. K. Sadozai, and S. Hakomori. 1990. Effect of lactose derivates on metastatic potential of B16 melanoma cells. Cancer Commun. 2:311-316.

33. Hakomori, S. 1991. Possible functions of tumor-associated carbohydrate antigens. Curr. Opin. in Immunol. 3:646-653.

34. Vollmers, H. P., and W. Birchmeier. 1983. Monoclonal antibodies inhibit the adhesion of mouse B16 melanoma cells in vitro and block lung metastasis in vivo. Proc. Natl. Acad. Sci. USA. 80:3729-3733.

35. Pierschbacher, M. D., and E. Ruoslahti. 1984. Cell attachment activity of fibronectin can be duplicated by small synthetic fragments of the molecule. $\mathrm{Na}$ ture (Lond.). 309:30-33.

36. Ruoslahti, E., and M. D. Pierschbacher. 1986. Arg-Gly-Asp-: a versatile cell recognition signal. Cell. 44:517-518.

37. Iwamoto, Y., F. A. Robey, J. Graf, M. Sasaki, H. K. Kleinman, Y. Yamada, and G. R. Martin. 1987. YIGSR, a synthetic laminin pentapeptide, inhibits experimental metastasis formation. Science (Wash. DC). 238:1132-1134.

38. Humphries, M. J. K. Olden, and K. M. Yamada. 1986. A synthetic peptide from fibronectin inhibits experimental metastasis of murine melanoma cells. Science (Wash. DC). 233:467-470.

39. Terranova, V. P., J. E. Williams, L. A. Liotta, and G. R. Martin. 1984 Modulation of the metastatic activity of melanoma cells by laminin and fibronectin. Science (Wash. DC). 226:982-985.

40. Lapis, K., S. Paku, and L. A. Liotta. 1988. Endothelialization of embolized tumor cells during metastasis formation. Clin. Exp. Metastasis. 6:73-90.

41. Weiss, L. 1987. The hemodynamic destruction of circulating cancer cells. Biorheology. 24:105-115.

42. Weiss, L., and D. S. Dimitrov. 1986. Mechanical aspects of the lungs as cancer cell-killing organs during hematogenous metastasis. J. Theor. Biol. 121:307-321.

43. Nicolson, G. L. 1988. Cancer metastasis: tumor cell and host organ properties important in metastasis to specific secondary sites. Biochim. Biophys. Acta. 948:175-224

44. Belloni, P. N., and R. J. Tressler. 1990. Microvascular endothelial cell heterogeneity: interactions with leukocytes and tumor cells. Cancer Metastasis Rev. 8:353-389.

45. Gunthert, U., M. Hofmann, W. Rudy, S. Reber, M. Zoller, I. Haussmann, S. Matzku, A. Wenzel, H. Ponta, and P. Herrlich. 1991. A new variant of glycoprotein CD44 confers metastatic potential to rat carcinoma cells. Cell. 65:13-24. 